KCL-PH-TH/2012-18, LCTS/2012-10, CERN-PH-TH/2012-103, TAUP-2949/12

\title{
Indications on the Mass of the Lightest Electroweak Baryon
}

\author{
John Ellis ${ }^{1,2}$ and Marek Karliner ${ }^{3}$ \\ ${ }^{1}$ Theoretical Particle Physics and Cosmology Group, Physics Department, \\ King's College London, London WC2R 2LS, UK \\ ${ }^{2}$ TH Division, Physics Department, CERN, CH-1211 Geneva 23, Switzerland \\ ${ }^{3}$ Raymond and Beverly Sackler School of Physics and Astronomy, Tel Aviv University, Israel
}

\begin{abstract}
In general, an effective low-energy Lagrangian model of composite electroweak symmetry breaking contains soliton solutions that may be identified with technibaryons. We recall how the masses of such states may be related to the coefficients of fourth-order terms in the effective Lagrangian, and review the qualitative success of this approach for baryons in QCD. We then show how the current theoretical and phenomenological constraints on the corresponding fourth-order coefficients in the electroweak theory could be used to estimate qualitative lower and upper bounds on the lightest electroweak baryon mass. We also discuss how the sensitivity of the LHC experiments could enable these bounds to be improved.
\end{abstract}

April 2012 


\section{Introduction}

One of the primary objectives of the LHC experimental programme is to explore the electroweak symmetry-breaking sector. Theoretical perspectives range between the possibility that there is a single elementary Higgs-like boson as in the Standard Model [1], and the possibility that electroweak symmetry is broken dynamically by a new strongly-interacting sector that might not yield a recognizable scalar boson, as in traditional technicolour models [2]. Intermediate possibilities include composite scenarios in which there is a relatively light pseudo-Nambu-Goldstone boson whose couplings might differ observably from those of a Standard Model Higgs boson [3].

The LHC experiments ATLAS [4] and CMS [5] currently exclude a Standard Model-like Higgs boson weighing $<122.5 \mathrm{GeV}$ and between $127.5 \mathrm{GeV}$ and $600 \mathrm{GeV}$. Open possibilities include a heavy Higgs-like boson as in traditional composite models, a (possibly elementary) Higgs-like boson with mass $125 \pm 2.5 \mathrm{GeV}$, and an intermediate-mass scalar boson with a smaller production cross section and/or observable decay branching ratios than a Standard Model Higgs boson. Present LHC data hint at the existence of a scalar boson weighing $\sim 125 \mathrm{GeV}$ [4,5], but this remains to be confirmed.

Independently from the possible existence of a recognizable scalar boson, there are already indications that any strongly-interacting electroweak symmetry-breaking sector could not simply be a scaled-up version of QCD. For example, QCD-like models would make excessive contributions to the electroweak vacuum polarization $S$ [6], and potentially also to $T$ [7]. For this reason, there is much discussion of 'walking technicolour' models [8] in which the coupling strength of the new strong interaction evolves relatively slowly over an extended range of energy scales, reflecting nearly-conformal dynamics. Such a scenario may also help suppress flavour-changing neutral interactions in extended technicolour models.

It is interesting to glean as much information as possible about the possibilities allowed for any such novel strongly-interacting sector. For example, in some such scenarios the effective low-energy spectrum contains a pseudo-dilaton [9, 10], a pseudo-Nambu-Goldstone boson of approximate conformal invariance. The above-mentioned LHC data constrain such a possibility over a wide range of possible pseudo-dilaton masses [10,11]. A complementary window on possible strongly-interacting electroweak symmetry-breaking sectors could be provided by technibaryons, the lightest of which would be stable in many models and therefore could be a candidate for cold dark matter [12].

Another window on the electroweak symmetry-breaking sector is provided by the search for non-standard interactions between massive vector bosons $V$, either indirectly via their 
effects on precision electroweak observables or directly via measurements of $V V$ scattering at the LHC. Lower limits on $V V$ interactions of fourth order in particle momenta (field derivatives) have been derived from crossing, Lorentz invariance and unitarity [13], which are considerably smaller than the upper limits that have been derived from precision electroweak data [14. It has also been argued that the LHC experiments should have the sensitivity to improve significantly on the current upper limits [14].

The purpose of this paper is to connect the technibaryon and $V V$ scattering windows on a possible strongly-interacting electroweak sector, using the Skyrme model of baryons [15, 16]. The second-order terms in the effective Lagrangian for electroweak symmetry breaking are isomorphic to the corresponding terms in the low-energy $\mathrm{SU}(2) \times \mathrm{SU}(2)$ chiral Lagrangian for QCD, which is known, in the presence of suitable generic fourth-order terms, to possess 'Skyrmion' soliton solutions that can be identified quite successfully with baryons [17, 18]. It is therefore natural also to use the Skyrme model to describe technibaryons, at least

qualitatively [10]. The properties of Skyrmions, in particular their masses, are related to the magnitudes of the fourth-order terms in the effective Lagrangian. Therefore, constraints on (measurements of) anomalous $V V$ scattering can be used to bound (estimate) the mass of the lightest technibaryon, a quantity of potential interest to astrophysical searches for dark matter as well as the LHC experiments.

We find that the present constraints on anomalous $V V$ scattering do not provide very strong constraints on the lightest technibaryon mass, though they might be of some interest for some walking technicolour models. On the other hand, LHC measurements of $V V$ scattering could provide much more restrictive information on the possible appearance of technibaryons in the multi-TeV range.

\section{Phenomenological Framework}

We work within the framework of the following nonlinear low-energy effective Lagrangian for the electroweak symmetry-breaking sector:

$$
\mathcal{L}_{e f f}=\frac{v^{2}}{4} \operatorname{Tr}\left(D_{\mu} U D^{\mu} U^{\dagger}\right)+\frac{1}{32 e^{2}} S+\ldots,
$$

where $U$ is a unitary $2 \times 2$ matrix parametrizing the three Nambu-Goldstone fields that are 'eaten' by the $W^{ \pm}$and $Z^{0}$, giving them masses, $v \sim 246 \mathrm{GeV}$ is the conventional electroweak scale, and the second, 'Skyrme term' [15, 16]

$$
S \equiv \operatorname{Tr}\left[\left(D_{\mu} U\right) U^{\dagger},\left(D_{\nu} U\right) U^{\dagger}\right]^{2}
$$


is scaled by an a priori unknown parameter $e$. The ... represent other possible terms of fourth and higher orders in a derivative expansion. The effective low-energy chiral Lagrangian for QCD has the same form as (1), with the electroweak scale $v$ replaced by the pion decay constant $f_{\pi} \sim 93 \mathrm{MeV}$.

The effective Lagrangian (1) necessarily has soliton solutions, because $\pi_{3}(\mathrm{SU}(2))=\mathrm{Z}$, which have integer baryon number

$$
B=\frac{1}{24 \pi^{2}} \int d^{3} x \epsilon^{i j k} \operatorname{Tr}\left[\left(U^{-1} \partial_{i} U\right)\left(U^{-1} \partial_{j} U\right)\left(U^{-1} \partial_{k} U\right)\right]
$$

In the case of a nonlinear $\mathrm{SU}(3) \times \mathrm{SU}(3) \rightarrow \mathrm{SU}(3)$ theory the effective Lagrangian contains a Wess-Zumino term $N \Gamma$ [19], where $N$ is an integer, and the lowest-lying $B=1$ baryon is a fermion (boson) with $I=J=\frac{1}{2}(I=J=0)$ if $N$ is odd (even). If the underlying stronglyinteracting theory is a non-Abelian $\mathrm{SU}(\mathrm{N})$ gauge theory with fermions, $N$ is identified with the number of colours, and in QCD the $B=1$ baryon is necessarily a fermion because $N=3$. In the case of the nonlinear $\mathrm{SU}(2) \times \mathrm{SU}(2) \rightarrow \mathrm{SU}(2)$ theory (1), there is no Wess-Zumino term, and the $B=1$ electroweak baryon may be either a boson or a fermion, with the latter remaining a topological possibility, since $\pi_{4}(\mathrm{SU}(2))=\mathrm{Z}_{2}$ [20, 21]. Here we leave open the question whether the electroweak baryonic 'Skyrmions' are bosons or fermions.

The masses and other properties of Skyrmions can be calculated in the semiclassical limit of large $N$. We consider such calculations to be quite successful in QCD, where $N=3$, and assume that they are qualitatively correct also in the electroweak sector. If the masses of the strongly-interacting fermions can be neglected, as assumed in technicolour models, numerical calculation of the lightest Skyrmion mass yields [16]

$$
M_{S} \sim \frac{73}{e} v+\ldots
$$

where the dots represent corrections due, e.g., to collective quantization of the rotational degrees of freedom in the fermionic case. In the case of QCD, we replace $v$ by $f_{\pi}$, and for the $I=J=\frac{1}{2}$ nucleon the rotational correction takes the form

$$
\Delta M_{S}=\frac{3}{8 \lambda}
$$

where $\lambda \sim(2 \pi / 3)\left(25.5 / e^{3} f_{\pi}\right)$, and the correction is a factor 5 larger in the case of the $I=J=\frac{3}{2} \Delta$ multiplet.

The fourth-order terms in the effective Lagrangian (1) are often parametrized as follows:

$$
\mathcal{L}_{\text {eff } 4}=\alpha_{4} \operatorname{Tr}\left[\left(V_{\mu} V^{\nu}\right)^{2}\right]+\alpha_{5} \operatorname{Tr}\left[\left(V_{\mu} V^{\mu}\right)^{2}\right]
$$


where $V_{\mu} \equiv\left(D_{\mu} U\right) U^{\dagger}$. The Skyrme term (2) is the antisymmetric combination $S=2\left\{\operatorname{Tr}\left[\left(V_{\mu} V^{\nu}\right)^{2}\right]-\operatorname{Tr}\left[\left(V_{\mu} V^{\mu}\right)^{2}\right]\right\}$, and we introduce the orthogonal, symmetric combination $T \equiv 2\left\{\operatorname{Tr}\left[\left(V_{\mu} V^{\nu}\right)^{2}\right]+\operatorname{Tr}\left[\left(V_{\mu} V^{\mu}\right)^{2}\right]\right\}$. Writing $\mathcal{L}_{\text {eff } 4} \equiv s \cdot S+t \cdot T$, we see that

$$
s=\frac{1}{32 e^{2}}=\frac{\alpha_{4}-\alpha_{5}}{4}, \quad t=\frac{\alpha_{4}+\alpha_{5}}{4} .
$$

and from eqs. (41) and (7), using $v=246 \mathrm{GeV}$ we find

$$
M_{S} \sim 102 \sqrt{s} \mathrm{TeV}
$$

In the following we use these relations and various theoretical and phenomenological bounds on $\alpha_{4,5}$ to constrain $e$ and hence estimate the mass of the lightest electroweak technibaryon within the Skyrmion framework.

\section{Bounds on Fourth-Order Chiral Lagrangian Terms and the QCD Skyrmion Mass}

In QCD a fit to the $I=J=\frac{1}{2}$ nucleon and the $I=J=\frac{3}{2} \Delta$ masses, assuming that their mass difference of $\sim 300 \mathrm{MeV}$ is provided by the semiclassical collective quantization of the rotational degree of freedom of the Skyrmion (5) and neglecting the light quark masses, yielded the value of $65 \mathrm{MeV}$ for $f_{\pi}$ (to be compared with the physical value $f_{\pi}=93 \mathrm{MeV}$ ) and $e=5.65$ [16]. Bearing in mind the discrepancy in $f_{\pi}$, this qualitative historical success suggests that the uncertainty in the estimate of the Skyrmion mass is $\sim \mathcal{O}(30) \%$.

Here we take a different approach, more similar to what we use later in the electroweak case, taking $f_{\pi}=93 \mathrm{MeV}$ from experiment and using data to estimate the magnitude of the Skyrme term in QCD. A global fit to the fourth-order terms in the effective low-energy Lagrangian of QCD [22] yields 23]

$$
\begin{aligned}
\alpha_{4} & =(1.4 \pm 0.3) \times 10^{-3}, \\
\alpha_{5}-\alpha_{4} & =(-2.7 \pm 1.3) \times 10^{-3},
\end{aligned}
$$

when these parameters are defined at a renormalization scale equal to $M_{\rho}$. These ranges are in good qualitative agreement with the predictions of the $1 / N_{c}$ expansion. Inserting them into (7) yields

$$
s=\frac{1}{32 e^{2}}=(0.7 \pm 0.3) \times 10^{-3}
$$

and hence $e=7_{-1}^{+3}$. 
This range lies somewhat above the value $e=5.45$ found in [16] from a combined fit to the nucleon and $\Delta$ masses, but we are encouraged that it is within a similar ballpark. Inserting this range into (44) and replacing $v$ by $f_{\pi}=93 \mathrm{MeV}$ yields the estimate $\sim 1000 \mathrm{MeV}$ for the nucleon mass, before incorporating the semiclassical correction (5) associated with collective quantization of the rotational degree of freedom. This is $\sim 220 \mathrm{MeV}$ for the central value $e \sim 7$, but is $\propto e^{3}$ and hence quite uncertain. Nevertheless, we are encouraged that the estimated nucleon mass is correct to within about $30 \%$, consistent with the expected uncertainty.

\section{Bounds on Fourth-Order Electroweak Terms and the Techni-Skyrmion Mass}

In the case of the electroweak theory, lower bounds on the coefficients $\alpha_{4,5}$ were obtained in [13], assuming just crossing, Lorentz invariance and unitarity:

$$
\begin{aligned}
\alpha_{4}(v) & >6 \times 10^{-4}, \\
\alpha_{4}(v)+\alpha_{5}(v) & >1.1 \times 10^{-3},
\end{aligned}
$$

where we have noted that $\alpha_{4,5}$ are specified at the scale of electroweak symmetry breaking [13. As might have been expected from the generality of the input assumptions, it is not possible to derive useful constraints on the technibaryon mass from (13). These constraints are clearly compatible with either $s \rightarrow 0$, in which case (8) would allow an arbitrarily small Skyrmion mass, or $s \rightarrow \infty$, in which case $M_{S}$ would be arbitrarily large. That said, one

might be tempted to consider the possibility that both the bounds (12, 13) were saturated, with the inference that

$$
s=\frac{1}{32 e^{2}} \sim 0.25 \times 10^{-4}, \quad t \sim 2.8 \times 10^{-4},
$$

corresponding naively to $e \sim 35$ and $M_{S} \sim 0.5 \mathrm{TeV}$. However, such an inference would be unsound, since $\alpha_{4,5}$ are subject to important renormalization effects [13]:

$$
\begin{aligned}
& \alpha_{4}(\mu)=\alpha_{4}(v)+\frac{1}{96 \pi^{2}} \ln \left(\frac{v}{\mu}\right), \\
& \alpha_{5}(\mu)=\alpha_{5}(v)+\frac{1}{192 \pi^{2}} \ln \left(\frac{v}{\mu}\right) .
\end{aligned}
$$

If one were to use $\mu \sim 500 \mathrm{GeV}$, one would find a reduction in $s$ by $\sim 0.9 \times 10^{-4}$, preventing the derivation of any estimated bound on $M_{S}$. 
Additional dynamical information is required if interesting bounds on $M_{S}$ are to be derived. For example, upper and lower limits on the magnitudes of the coefficients $\alpha_{4,5}$ were obtained in [14, on the basis of precision electroweak data:

$$
\begin{aligned}
& -0.35<\alpha_{4}(v)<0.06 \\
& -0.87<\alpha_{5}(v)<0.15 .
\end{aligned}
$$

These are compatible with $\alpha_{4}=\alpha_{5}$ and hence $s=0$, and so not provide a lower bound on the electroweak baryon mass. However, an upper bound on $M_{S}$ can be obtained by substituting $\alpha_{4}<0.06$ and $\alpha_{5}>-0.87$ into (7), obtaining $s<0.23$, and hence

$$
M_{S}<50 \mathrm{TeV}
$$

This constraint is quite weak, though it might be relevant in the context of some models of 'walking technicolour'.

It was also estimated in [14 that non-observation of $\alpha_{4,5}$ by the LHC experiments ATLAS and CMS would restrict these coefficients to the ranges

$$
\begin{aligned}
-7.7 \times 10^{-3}<\alpha_{4}(v)<15 \times 10^{-3} \\
-12 \times 10^{-3}<\alpha_{5}(v)<10 \times 10^{-3} .
\end{aligned}
$$

If $\alpha_{4,5}$ were indeed not to be measured at these levels, one could conclude that $s<7 \times 10^{-3}$ and $e>2.1$, hence

$$
M_{S} \lesssim 8.5 \mathrm{TeV}
$$

We note that both the bounds (18, 20) are reasonably insensitive to the inclusion of the renormalization effects (15, 16), which would change $\alpha_{4,5}$ by $\sim 4 \times 10^{-3}, 2 \times 10^{-3}$ even if one chose $\mu=10 \mathrm{TeV}$. The possible future constraint (20) is much stronger than the present bound (18), and may be of relevance to a wider class of technicolour models.

We have neglected in this Section the semiclassical correction to the Skyrmion mass due to collective quantization of the rotational degrees of freedom (5), that appears if the technibaryon is a fermion but would be absent if it is a boson. This may be estimated on the basis of (5) to be $\Delta M \approx 7 \times 10^{-3} e^{3} v=9.5 / s^{3 / 2} \mathrm{MeV}$, corresponding to $\approx 16 \mathrm{GeV}$ if the bound (19) is saturated. In fact, as argued in [10], there are at least two reasons why the technibaryon should be a boson, at least if it is stable on a cosmological time scale. One is that the cold dark matter scattering cross section of a fermionic technibaryon is likely to exceed the experimental upper limit [24]. The other is that, although the lightest fermionic technibaryon might well be electromagnetically neutral and stable, its nominally 
unstable charged partner might form stable charged techninuclei 1, whose abundance could exceed the experimental upper limits on charged relics from the Big Bang [10]. Thus the rotational correction (5) to the techniSkyrmion mass is likely to be irrelevant. Nevertheless, the semiclassical mass estimates made here should be treated with caution.

\section{Relation to Vector-Meson Parameters}

Before closing, we recall that a connection may exist between the fourth-order Lagrangian coefficients and the masses $M_{V_{i}}$ and couplings $G_{V_{i}}$ of vector mesons. In the framework of resonance chiral theory [22], one finds [23]

$$
\alpha_{4}=2 \alpha_{5}=\frac{G_{V}^{2}}{4 M_{V}^{2}}
$$

where in a single-meson dominance approximation it is estimated that $G_{V}=v / \sqrt{2}$. These relations can be combined with the constraints (17, 19) to derive present bounds on and prospective LHC sensitivities to $M_{V}$ in the single-resonance approximation. In both cases, we find that the constraint on $\alpha_{4}$ is more powerful, yielding

$$
\begin{aligned}
\text { Present bound : } & M_{V}>350 \mathrm{GeV}, \\
\text { Future sensitivity : } & M_{V}>700 \mathrm{GeV} .
\end{aligned}
$$

We recall that the corresponding relations in QCD are qualitatively successful at the $\sim 30 \%$ level [23]. However, much as the QCD analogy may not apply to the calculation of the $S$ parameter, the single-resonance approximation may not be valid in the class of stronglyinteracting electroweak models of interest [8].

\section{Discussion}

In this paper we have explored the connection between the possible mass of the lightest electroweak baryon (technibaryon) and fourth-order terms in the low-energy expansion of the effective Lagrangian for electroweak symmetry breaking that is provided by the Skyrme model. Fundamental considerations based on crossing, Lorentz invariance and unitarity [13] do not provide very stringent constraints on the possible electroweak baryon mass, nor do present electroweak data [14]. However, a significant improvement in the indicative upper limit could be provided by LHC data [14, and further improvement could presumably be

\footnotetext{
${ }^{1}$ Much as the unstable neutron is present in the Universe today in stable nuclei.
} 
obtained using the more precise measurements of $V V$ scattering that would become possible at a high-energy $e^{+} e^{-}$collider.

It is an interesting question whether measurements of $V V$ scattering combined with other experiments could eventually overconstrain or exclude a strongly-interacting electroweak sector of the type discussed here. In this respect, an important issue is whether the lightest electroweak baryon is stable, or not. We recall that anomalous electroweak interactions could, in principle, cause the lightest electroweak baryon to decay [25] into final states involving all the electroweak doublets of the Standard Model, depending on the structure of the new strongly-interacting sector. The lifetime of the lightest electroweak baryon could in principle be very short in some models, if its mass exceeds a certain critical value [25].

If the lightest electroweak baryon is stable on a cosmological time scale, dark matter scattering experiments have the potential to detect or exclude a massive neutral electroweak baryon [10,24]. In addition, bound states of neutral and charged electroweak baryons might form detectable, massive 'techninuclei' [10]. If there is a more massive charged state with a lifetime exceeding a nanosecond, it could be detected in a collider experiment. Electroweak baryons with shorter lifetimes could be detected through their decays into a plethora of Standard Model particles.

The possible phenomenology of electroweak baryons is very rich, and depends on their possible masses. As we have pointed out in this paper, there is a possible connection with other electroweak observables that will surely repay further study.

\section{Acknowledgements}

This study was triggered during the SCGT12 MiniWorkshop at the Kobayashi-Maskawa Institute in Nagoya, and J.E. thanks K. Yamawaki and his colleagues for hospitality there. We thank A. Pich for helpful correspondence. The work of J.E. was supported partly by the London Centre for Terauniverse Studies (LCTS), using funding from the European Research Council via the Advanced Investigator Grant 267352.

\section{References}

[1] F. Englert and R. Brout, Phys. Rev. Lett. 13 (1964) 321; P. W. Higgs, Phys. Rev. Lett. 13 (1964) 508; P. W. Higgs, Phys. Lett. 12 (1964) 132; G. S. Guralnik, C. R. Hagen and T. W. B. Kibble, Phys. Rev. Lett. 13 (1964) 585; P. W. Higgs, Phys. Rev. 145 (1966) 1156; T. W. B. Kibble, Phys. Rev. 155 (1967) 1554. 
[2] S. Weinberg, Phys. Rev. D 13 (1976) 974; L. Susskind, Phys. Rev. D 20 (1979) 2619.

[3] G. F. Giudice, C. Grojean, A. Pomarol and R. Rattazzi, JHEP 0706 (2007) 045 hep-ph/0703164]; R. Contino, C. Grojean, M. Moretti, F. Piccinini and R. Rattazzi, JHEP 1005 (2010) 089 [arXiv:1002.1011 [hep-ph]]; R. Contino, arXiv:1005.4269 [hepph]; R. Grober and M. Muhlleitner, JHEP 1106 (2011) 020 [arXiv:1012.1562 [hep-ph]].

[4] G. Aad et al. [ATLAS Collaboration], Phys. Lett. B 710 (2012) 49 arXiv:1202.1408 [hep-ex]].

[5] S. Chatrchyan et al. [CMS Collaboration], Phys. Lett. B 710 (2012) 26 [arXiv:1202.1488 [hep-ex]].

[6] M. E. Peskin and T. Takeuchi, Phys. Rev. D 46 (1992) 381.

[7] R. S. Chivukula, Phys. Rev. Lett. 61 (1988) 2657.

[8] B. Holdom, Phys. Lett. B 150 (1985) 301; K. Yamawaki, M. Bando and K. i. Matumoto, Phys. Rev. Lett. 56 (1986) 1335; T. W. Appelquist, D. Karabali and L. C. R. Wijewardhana, Phys. Rev. Lett. 57 (1986) 957; T. Akiba and T. Yanagida, Phys. Lett. B 169 (1986) 432.

[9] W. D. Goldberger, B. Grinstein and W. Skiba, Phys. Rev. Lett. 100 (2008) 111802 arXiv:0708.1463 [hep-ph]]; J. Fan, W. D. Goldberger, A. Ross and W. Skiba, Phys. Rev. D 79 (2009) 035017 [arXiv:0803.2040 [hep-ph]]; L. Vecchi, Phys. Rev. D 82 (2010) 076009; B. Grinstein and P. Uttayarat, JHEP 1107 (2011) 038 arXiv:1105.2370 [hepph]]; V. Barger, M. Ishida and W. Keung, arXiv:1111.2580 [hep-ph]; B. Coleppa, T. Gregoire and H. E. Logan, arXiv::1111.3276 [hep-ph].

[10] B. A. Campbell, J. Ellis and K. A. Olive, JHEP 1203 (2012) 026 arXiv:1111.4495 [hep-ph]].

[11] A. Azatov, R. Contino and J. Galloway, arXiv:1202.3415 [hep-ph]; J.R. Espinosa, C. Grojean, M. Muhlleitner and M. Trott, arXiv:1202.3697 [hep-ph]; P. P. Giardino, K. Kannike, M. Raidal and A. Strumia, arXiv:1203.4254 [hep-ph]; T. Li, X. Wan, Y. Wang and S. Zhu, arXiv:1203.5083 [hep-ph]; M. Rauch, arXiv:1203.6826 [hep-ph]; J. Ellis and T. You, arXiv:1204.0464 [hep-ph].

[12] S. Nussinov, Phys. Lett. B 165 (1985) 55. 
[13] J. Distler, B. Grinstein, R. A. Porto and I. Z. Rothstein, Phys. Rev. Lett. 98 (2007) 041601 arXiv:hep-ph/0604255.

[14] O. J. P. Eboli, M. C. Gonzalez-Garcia and J. K. Mizukoshi, Phys. Rev. D 74 (2006) 073005 arXiv:hep-ph/0606118.

[15] T. H. R. Skyrme, Proc. Roy. Soc. Lond. A 260, 127 (1961); Nucl. Phys. 31 (1962) 556.

[16] G. S. Adkins, C. R. Nappi and E. Witten, Nucl. Phys. B 228, 552 (1983).

[17] G. S. Adkins and C. R. Nappi, Nucl. Phys. B 233 (1984) 109; Nucl. Phys. B 249 (1985) 507; M. Karliner and M. P. Mattis, Phys. Rev. Lett. 56 (1986) 428; M. P. Mattis and M. Karliner, Phys. Rev. D 31 (1985) 2833; M. Karliner and M. P. Mattis, Phys. Rev. D 34 (1986) 1991.

[18] H. Weigel, Lect. Notes Phys. 743 (2008) 1.

[19] J. Wess and B. Zumino, Phys. Lett. B 37, 95 (1971).

[20] D. Finkelstein and J. Rubinstein, J. Math. Phys. 9, 1762 (1968).

[21] E. Witten, Nucl. Phys. B 223, 433 (1983).

[22] G. Ecker, J. Gasser, A. Pich and E. de Rafael, Nucl. Phys. B 321 (1989) 311.

[23] A. Pich, PoS Confinement 8 (2008) 026 arXiv:0812.2631 [hep-ph]].

[24] J. Bagnasco, M. Dine and S. D. Thomas, Phys. Lett. B 320 (1994) 99 arXiv:hep-ph/9310290.

[25] V. A. Rubakov, B. E. Stern and P. G. Tinyakov, Phys. Lett. B 160 (1985) 292. 